\title{
Cognitive decline is related to high blood glucose levels in older Chinese adults with the ApoE $\varepsilon 3 / \varepsilon 3$ genotype
}

\author{
Qi Qiư, Xiang Lin ${ }^{\dagger}$, Lin Sun, Min-jie Zhu, Tao Wang, Jing-hua Wang, Guan-jun Li, Shi-fu Xiao ${ }^{*}$ and Xia Li
}

\begin{abstract}
Background: Few studies have investigated the effects of blood glucose (BG) on cognitive function in communitydwelling elderly individuals carrying the apolipoprotein E (APOE) $\varepsilon 3$ allele.

Objective: To explore the effect of high BG levels on cognitive function in APOE $\varepsilon 3$-carrying, non-demented, community-dwelling older adults, as compared to their counterparts carrying the APOE $\varepsilon 4$ or APOE $\varepsilon 2$ alleles.

Methods: Within the China Longitudinal Ageing Study, we recruited 282 elderly adults without dementia. Data collected included demographic information; psychological measures; laboratory test results, including BG and plasma lipid levels; and APOE genotypes. We divided the participants into APOE $\varepsilon 2(\varepsilon 2 / \varepsilon 2, \varepsilon 2 / \varepsilon 3), \varepsilon 3(\varepsilon 3 / \varepsilon 3)$, and $\varepsilon 4(\varepsilon 3 / \varepsilon 4, \varepsilon 4 / \varepsilon 4)$ groups. Partial correlation analyses and multivariate linear regression analyses were utilized to compare the cognitive function and laboratory data between the APOE groups. White matter hyperintensity (WMH) was measured on magnetic resonance images in 77 participants.

Results: With adjustment for age, sex, education, and diabetes, higher BG in non-demented community-dwelling older adults was associated with cognitive decline in immediate memory and executive function. In the APOE $\varepsilon 3$ group, elevated BG was associated with cognitive decline in immediate memory, executive function, and perceptual reasoning. In the APOE $\varepsilon 4$ group, higher BG was also correlated with a decline in abstract reasoning. There was a trend for association between higher BG and more severe WMHs.
\end{abstract}

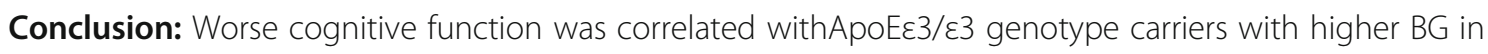
community-dwelling older adults.

Keywords: Apolipoproteins E, Cognition, Blood glucose, Aged, White matter, Community-based

\section{Background}

As the world population ages, the number of people living with cognitive disease is rising rapidly. The World Alzheimer Report 2018 reports that the number of people living with dementia is likely to triple from 50 million to 152 million by 2050 [1]. Most of the affected individuals are from middle and lower income countries [1]. The associated cognitive impairment leads to a high rate of disability and generates a heavy economic burden.

\footnotetext{
* Correspondence: xiaoshifu@msn.com; lixia11111@alumni.sjtu.edu.cn Qi Qiu and Xiang Lin are co-first authors on this work

Department of Psychogeriatrics, Shanghai Mental Health Center, Shanghai Jiao Tong University School of Medicine, South WanPing Road 600, Shanghai 200030, China
}

Many clinical and population-based studies suggest cognitive function is associated with high blood levels of total cholesterol (TC), low-density lipoprotein cholesterol (LDL-C), triglycerides (TG), glucose, and low levels of high -density lipoprotein cholesterol (HDL-C) [2, 3]. Gill Livingston and her colleagues have suggested that diabetes is a modifiable risk factor of dementia [4]. A study that investigated the Chinese Alzheimer's disease (AD) population found that high normal fasting blood glucose (BG) level was associated with dementia, independently of vascular risk factors [5]. Moreover, among dementia-free older adults, those with high BG also had poorer overall cognitive performance [6]. The toxicity of hyperglycemia might mediate microvascular

(c) The Author(s). 2019 Open Access This article is distributed under the terms of the Creative Commons Attribution 4.0 International License (http://creativecommons.org/licenses/by/4.0/), which permits unrestricted use, distribution, and reproduction in any medium, provided you give appropriate credit to the original author(s) and the source, provide a link to the Creative Commons license, and indicate if changes were made. The Creative Commons Public Domain Dedication waiver (http://creativecommons.org/publicdomain/zero/1.0/) applies to the data made available in this article, unless otherwise stated. 
abnormalities and may be associated with greater white matter lesion volumes $[7,8]$.

A well-established susceptibility genetic factor for Alzheimer's disease is the apolipoprotein $\mathrm{E}$ (APOE) $\varepsilon 4$ allele [9]. APOE in the central nervous system is key to the uptake of lipids and their reallocation to cells for myelin generation or membrane repair [10]. APOE $\varepsilon 4$ preferentially combines large lipoprotein particles and is associated with an increased risk for atherosclerosis [11] and vascular dementia [12]. APOE has been shown to stimulate $A \beta$ production in human neurons with an APOE 4>APOE3 > APOE2 potency rank order [13]. Some drugs affect cholesterol metabolism through mechanisms that probably induce APOE synthesis [14]. In peripheral blood studies, APOE $\varepsilon 4$ was shown to be associated with higher total cholesterol and higher LDL-C levels, whereas APOE $\varepsilon 2$ was associated with lower levels of these markers [15]. But in our previous study, APOE $\varepsilon 3 / \varepsilon 3$ genotype carriers among community-dwelling elderly individuals exhibited higher BG [16]. Some researchers have reported that diabetes and the APOE $\varepsilon 4$ allele interact to impact cognitive function [17]. Diabetes along with the APOE $\varepsilon 4$ allele might cause neuronal and vessel damage, increasing the risk for $\mathrm{AD}$ or mixed dementias $[18,19]$. APOE $\varepsilon 3 / \varepsilon 3$ is the most frequent genotype and considered the neutral risk genotype. Few studies have investigated the correlation between cognitive function and BG or lipid profile in APOE $\varepsilon 3 / \varepsilon 3$ genotype carriers independently. Therefore, this study investigated the association between cognitive performance and both BG and the lipid profile in APOE $\varepsilon 3 / \varepsilon 3$ genotype carriers, as compared to that in APOE $\varepsilon 4$ or APOE $\varepsilon 2$ allele carriers, in dementia-free, communitydwelling older Chinese Han adults.

\section{Methods}

\section{Sample and design}

This study involved random sampling of communitydwelling elderly individuals (over 60 years old) from Changning district and Pudong new district from 2011 to 2012. We included 283 participants without dementia. Twenty-one elderly individuals with dementia were excluded, among whom five participants were APOE $\varepsilon 4$ carriers and fifteen were APOE $\varepsilon 3 / \varepsilon 3$ carriers. The inclusion criteria were age of 60 years or older with normal audio-visual function after correction. Exclusion criteria were as follows: the participants had diagnosed dementia; a major psychiatric disorder, such as schizophrenia; or a systemic illness that affected their ability to complete the assessment.

\section{Procedures}

This survey used the China Longitudinal Ageing Study (CLAS) protocol [20]. Data collected included general demographic information, standard psychometric scales, APOE genotyping, and laboratory test results.

APOE genotyping was based on allele-specific polymerase chain reaction (PCR) methodology adapted to real-time PCR using a TaqMan probe [21]. The laboratory tests included fasting BG, TC, TG, LDL-C, and HDL-C level assessments. Cognitive function was measured using the Beijing version of the Montreal Cognitive Assessment (MoCA) [22], and the Neuropsychological Test Battery (NTB) [23], administered by a psychologist. Attending-level psychiatrists collected information on the current state and history of disease, conducted physical examinations, and determined a diagnosis using the Structured Clinical Interview for DSM-IV (SCID).

Seventy-seven participants underwent magnetic resonance imaging (MRI) and white matter lesion hyperintensity (WMH) was evaluated using the Fazekas scale [24]. The scale is composed of two parts, the periventricular lesion hyperintensity (PWMH) and deep white matter lesion hyperintensity (DWMH). Each part was scored, with a score ranging from 0 to 3 ; thus, the total score ranged from 0 to 6 , with a higher score indicating more serious lesions.

All the subjects had signed an informed consent at the start of the study, and ethical approval was obtained from Shanghai Mental Health Center.

\section{Cognitive function assessment}

The Beijing version of MoCA is a brief cognitive screening tool for mild cognitive impairment. It includes executive function, attention, language, visuospatial and orientation, and was used to assess global cognitive function.

The NTB consists of the following seven components: 1) the Wechsler Memory-Digit Span (WMDS, score range 0 to 24), including the Digits Forward and Digits Backward section where participants repeat a forward and backward sequence of numbers, and each sequence of numbers has more digits than the last; 2) the Rey Auditory Verbal Learning-Immediate test (RAVL-I, 0 to 75), where participants are given a list of 15 unrelated words repeated over five different trials and are asked to repeat them; 3) the Wechsler Memory Visual-Immediate test (WMVis-I, 0 to 18), which includes functional and semantic connections (matching pictures belonging to one category), recognition (reconfirm whether the pictures appeared before or not), and visual matching and abstracting (select picture items according to certain rules); 4) the Controlled Word Association Test (COWAT) which assessed the spontaneous production of words belonging to the category "vegetable", and combinations of words with the Chinese words "shui" and "fa"; 5) the Rey Auditory Verbal Learning Delayed test (RAVL-D, 0 to 45 ) in which another list of 15 
unrelated words was given and the participants had to repeat the original list of 15 words, and then repeat them again after $30 \mathrm{~min}$; 6) the Wechsler Adults Intelligence Scale - Picture Completion test (WAIS-PC, 0 to 21), in which participants had to specify certain absent portions of drawings of familiar items; and 7) the Wechsler Adults Intelligence Scale - Block Design (WAIS-BD, 0 to 48) where the participants used hand movements to rearrange blocks that had various color patterns on different sides to match a given pattern. The NTB was used to assess immediate memory (WMVis-I and RAVL-I), delayed memory (RAVL-D), executive function (WMDS and COWAT), and perceptual reasoning (WAIS-PC and WAIS-BD).

\section{Statistical analyses}

The genotype groups were divided into APOE $\varepsilon 2$ (APOE $\varepsilon 2 / \varepsilon 3$ and $\varepsilon 2 / \varepsilon 2)$, APOE $\varepsilon 3(\varepsilon 3 / \varepsilon 3)$, and APOE $\varepsilon 4(\varepsilon 2 / \varepsilon 4$, $\varepsilon 3 / \varepsilon 4, \varepsilon 4 / \varepsilon 4)$. Comparisons in the three groups were performed with an analysis of variance for continuous variables, and post hoc analyses were done between each set of two groups. The $\chi^{2}$ test was used for dichotomous variables. BG, MoCA score, and HDL had non-normal distributions, so a non-parametric test was used. Partial correlation analyses were performed to evaluate associations among glucose, lipid profiles, and cognitive function, with adjustment for education, age, sex, and diabetes, in the different APOE groups. Dependent variables were the $\mathrm{z}$-score of the MoCA and NTB scores. The independent variables were APOE genotype and laboratory test results. Multivariate linear regression analyses were used to examine the relationships between cognition, age, education, and laboratory data. As not all subjects underwent MRI (54 in the APOE $\varepsilon 3,12$ in the APOE $\varepsilon 4$, and 11 in the APOE $\varepsilon 2$ groups), we matched 12 participants in terms of age and sex between the normal $(<6.1 \mathrm{mmol} / \mathrm{L})$ and abnormal $(\geq 6.2 \mathrm{mmol} / \mathrm{L})$ BG groups in the APOE $\varepsilon 3$ group. We used the $t$-test to compare WMHs in these two BG groups, adjusting for education. Significance levels of 0.05 were used for all the tests. All statistical analyses were performed using SPSS version 21.0 (IBM Corp., Armonk, NY, USA).

\section{Results}

\section{Demographic characteristics}

This study was a cross-sectional study of CLAS and included 283 elderly people without dementia. The mean age at investigation was 71.63 years $( \pm 8.21$ years $)$ and mean years of education was $8.78( \pm 4.53)$; 169 (59.7\%) participants were female, and 47 (16.6\%) were APOE $\varepsilon 4$ carriers. The mean MoCA score was 22.29 (5.77). Demographic information is shown in Table 1.

The participants in the APOE $\varepsilon 3$ group were older than those in the APOE $\varepsilon 4$ group $(p=0.044)$. Compared to those in the APOE $\varepsilon 3$ and APOE $\varepsilon 4$ groups, the individuals in the APOE $\varepsilon 2$ group had less education ( $p=$ $0.019, p=0.009$, respectively). The BG in the APOE $\varepsilon 2$ group was lower than that in the APOE $\varepsilon 3$ group $(p=$ 0.045). LDL-C levels in the APOE $\varepsilon 2$ group were lower than in the APOE $\varepsilon 3$ and APOE $\varepsilon 4$ groups $(p=0.020, p$ $=0.018$, respectively). The HDL-C in the APOE $\varepsilon 2$ was higher than in the APOE $\varepsilon 4$ group $(p=0.037)$. The MoCA scores and the current state and history of disease, including hypertension, diabetes mellitus, and

Table 1 Demographic information in the total sample and different APOE groups

\begin{tabular}{|c|c|c|c|c|c|c|c|}
\hline & $\begin{array}{l}\text { Total } \\
(N=283)\end{array}$ & $\begin{array}{l}\text { APOE } \varepsilon 2 \\
(N=39)\end{array}$ & $\begin{array}{l}\text { APOE } \varepsilon 3 \\
(N=197)\end{array}$ & $\begin{array}{l}\text { APOE } \varepsilon 4 \\
(N=47)\end{array}$ & $\begin{array}{l}\varepsilon 2 \text { Vs } \varepsilon 3 \\
p\end{array}$ & $\varepsilon 2$ vs $\varepsilon 4$ & $\varepsilon 3$ vs $\varepsilon 4$ \\
\hline Age (years) & $71.63(8.22)$ & $71.64(8.16)$ & $72.14(8.44)$ & $69.45(7.06)$ & 0.727 & 0.217 & 0.044 \\
\hline Education (years) & $8.78(4.53)$ & $8.13(4.24)$ & $8.51(4.69)$ & $10.43(3.70)$ & 0.625 & 0.019 & 0.009 \\
\hline $\mathrm{BMI}\left(\mathrm{kg} / \mathrm{m}^{2}\right)$ & $24.02(3.36)$ & $24.09(3.35)$ & $23.97(3.39)$ & $24.20(3.28)$ & 0.834 & 0.890 & 0.676 \\
\hline Female & 169 (59.7\%) & $25(64.1 \%)$ & 118 (59.9\%) & $26(55.3 \%)$ & 0.707 & & \\
\hline $\mathrm{BG}(\mathrm{mmol} / \mathrm{L})$ & $5.70(2.00)$ & 5.15 (0.98) & $5.85(2.23)$ & $5.55(1.44)$ & 0.006 & 0.338 & 0.591 \\
\hline $\mathrm{TC}(\mathrm{mmol} / \mathrm{L})$ & $4.86(1.10)$ & $4.73(1.10)$ & $4.87(1.14)$ & $4.93(0.95)$ & 0.454 & 0.395 & 0.744 \\
\hline $\mathrm{TG}(\mathrm{mmol} / \mathrm{L})$ & $1.83(1.31)$ & $1.86(0.92)$ & $1.80(1.25)$ & 1.95 (1.74) & 0.773 & 0.754 & 0.466 \\
\hline $\mathrm{LDL}-\mathrm{C}(\mathrm{mmol} / \mathrm{L})$ & $2.90(0.90)$ & $2.57(0.87)$ & $2.94(0.93)$ & $3.03(0.75)$ & 0.020 & 0.018 & 0.516 \\
\hline $\mathrm{HDL}-\mathrm{C}(\mathrm{mmol} / \mathrm{L})$ & $1.17(0.27)$ & $1.21(0.32)$ & $1.18(0.27)$ & $1.09(0.22)$ & 0.872 & 0.138 & 0.077 \\
\hline Hypertensive & 151 (53.5\%) & $23(60.5 \%)$ & 104 (52.8\%) & $24(51.15 \%)$ & 0.636 & & \\
\hline Diabetes mellitus & 45 (15.3\%) & $3(7.9 \%)$ & $35(17.8 \%)$ & 7 (14.9\%) & 0.316 & & \\
\hline Hyperlipidemia & $52(19.8 \%)$ & $9(23.7 \%)$ & 36 (19.9\%) & 7 (15.9\%) & 0.084 & & \\
\hline MoCA & $22.29(5.77)$ & $22.10(5.31)$ & $21.94(6.02)$ & $23.91(4.81)$ & 0.998 & 0.281 & 0.055 \\
\hline
\end{tabular}

$B M I$ body mass index, $B G$ blood glucose, $T C$ total cholesterol, $T G$ triglycerides, $L D L-C$ low destiny lipoprotein cholesterol, $H D L-C$ high destiny lipoprotein cholesterol, MoCA Montreal Cognitive Assessment

Data are shown as mean (SD) or number (percentage) 
hyperlipidemia, were not different among the APOE groups. Overall, 28 of 52 patients with hyperlipidemia and 36 of 45 people with diabetes were treated with drugs.

\section{Relationship between blood glucose and cognitive function}

Six participants had missing NTB data. The partial correlation analysis between cognitive function and independent variables (glucose, lipid data) indicated that BG was related to RAVL-I, COWAT, RAVL-D scores, with adjustment for age, sex, education, and diabetes (Table 2). HDL was related to the WAIS-BD $(r=0.140, p=0.025)$.

Seventy-eight participants (27.6\%) with BG greater than $6.1 \mathrm{mmol} / \mathrm{L}$ were considered to be the abnormal glucose group. The z-scores of the cognitive scores between the normal and abnormal glucose groups were compared. Figure 1 shows the results after controlling for sex, age, education, and diabetes. There were significant differences in the RAVL-I $(p=0.020)$ and COWAT $(p=0.022)$ scores between the abnormal and normal glucose groups.

\section{Relationship between blood glucose, lipid profile, and cognitive performance in different APOE groups}

The partial correlation analysis revealed that, in the APOE $\varepsilon 3$ group, BG was negatively correlated with the cognitive scores from the RAVL-I $(r=-0.200, p=0.005)$, COWAT $(r=-0.169, p=0.019)$, RAVL-D $(r=-0.144, p=0.040)$, and WAIS-BD $(r=-0.145, p=0.045)$ tests. HDL was positively correlated with WMDS $(r=0.172, p=0.017)$ and WAIS-BD $(r=0.203, p=0.005)$ scores, and LDL was negatively correlated with MoCA scores $(r=-0.145, p=0.044)$. In the APOE $\varepsilon 4$ group, triglycerides were negatively correlated with WMVis-I scores $(r=-0.342, p=0.025)$, and positively correlated with the WAIS-BD scores $(r=0.315$,

Table 2 Partial correlation between glucose and cognitive scores

\begin{tabular}{lll}
\hline & \multicolumn{2}{l}{ Blood glucose } \\
\cline { 2 - 3 }$R$ & -0.064 & $p$ \\
\hline MoCA & 0.019 & 0.285 \\
WMDS & -0.168 & 0.750 \\
RAVL-I & -0.064 & 0.005 \\
WMV is-I & -0.149 & 0.285 \\
COWAT & -0.140 & 0.013 \\
RAVL-D & -0.086 & 0.019 \\
WAIS-PC & -0.085 & 0.155 \\
WAIS-BD & & 0.156 \\
\hline
\end{tabular}

MoCA Montreal Cognitive Assessment, WMDS Wechsler Memory-Digit Span, RAVL-I Rey Auditory Verbal Learning-Immediate, WMVis-I Wechsler Memory Visual-Immediate, COWAT Controlled Word Association Test, RAVL-D Rey Auditory Verbal Learning-Delayed, WAIS-PC Wechsler Adults Intelligence ScalePicture Completion, WAIS-BD Wechsler Adults Intelligence Scale-Block Design

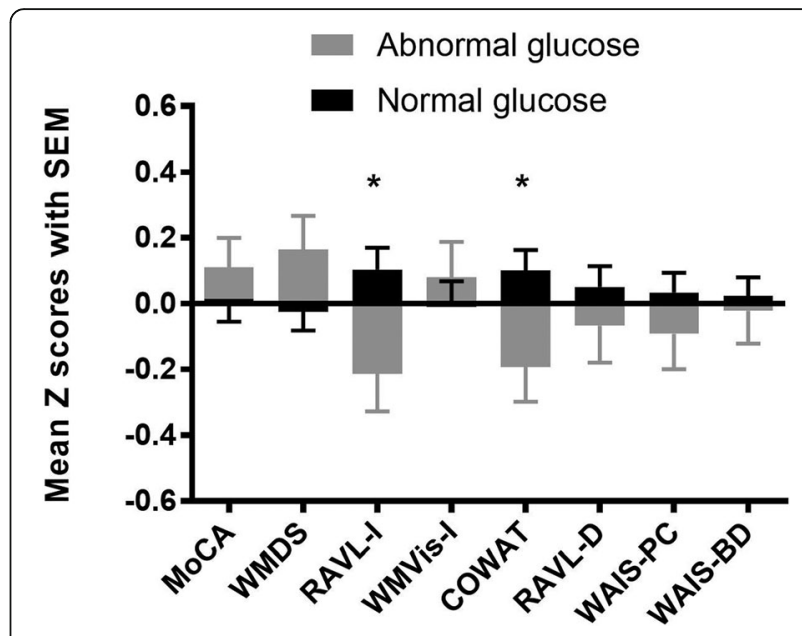

Fig. 1 Differences in cognitive function between different BG groups, adjusting for age, sex, education, and diabetes. There were significant differences in the RAVL-I $(p=0.024)$ and COWAT $(p=0.022)$ scores between the abnormal and normal glucose groups. ${ }^{*} p<0.05$

$p=0.039)$. In the APOE $\varepsilon 2$ group, there was no association between cognitive function and BG or lipid data.

In stepwise regression analyses in the different APOE groups, we used the MoCA, WMDS, RAVL-I, WMVis-I, COWAT, RAVL-D, and WAIS-BD scores as dependent variables, and the demographic variables, BG, and lipid data as independent variables. In the APOE $\varepsilon 3$ group, BG was significantly associated with RAVL-I $(\beta=-$ $0.141, p=0.037)$, COWAT $(\beta=-0.140, p=0.023)$, and WAIS-BD $(\beta=-0.173, p=0.005)$ scores, and HDL was positive associated with WMDS $(\beta=-0.146, p=0.011)$ scores. In the APOE $\varepsilon 4$ group, higher BG was associated with worse performance on the WMVis-I test $(\beta=-$ $0.301, p=0.023)$.

\section{White matter hyperintensities in different APOE $\varepsilon 3-$ carrying BG groups}

There were 12 APOE $\varepsilon 3$ carriers with abnormal BG, for whom WMH scores were available. We matched these with another 12 APOE $\varepsilon 3$ carriers with normal BG in terms of age and sex. Eight participants in the abnormal BG group had a hypertension history, as compared to three in the normal BG group. We compared the periventricular WMH scores, deep WMH score, and total WMH score in the two BG groups, with adjustment for education and hypertension history. These results are shown in Fig. 2. The differences in the total WMH between the two groups approached significance $(t=-2.065, p=0.052)$.

\section{Discussion}

In this community-based, cross-sectional study, we found that cognitive decline in terms of immediate memory and executive function was associated with 


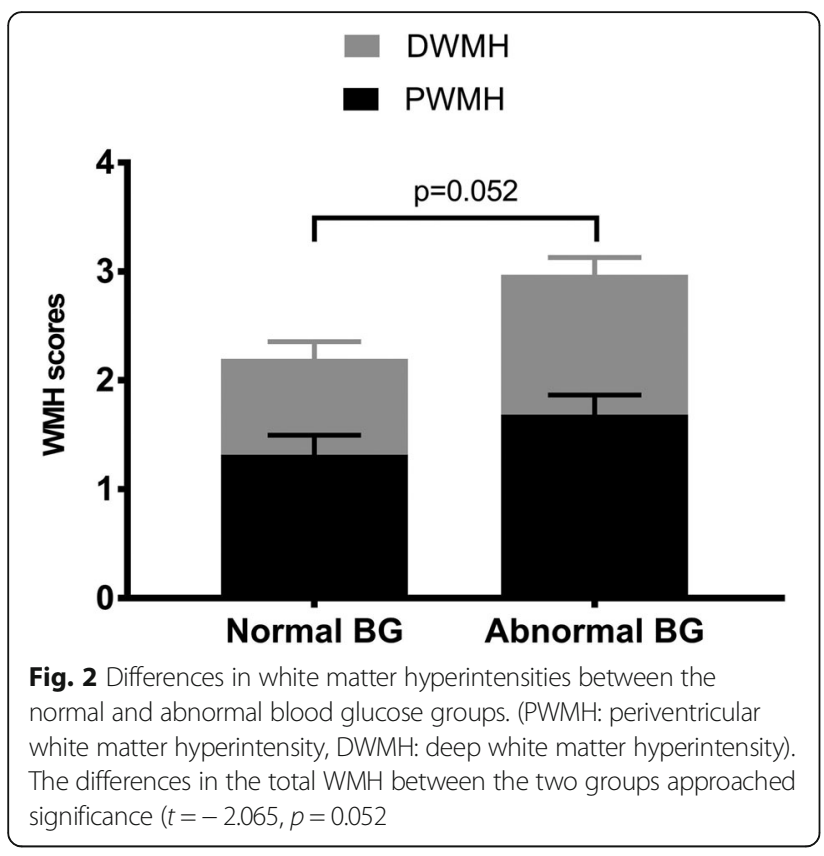

higher BG in non-demented, community-dwelling older adults. Perceptual reasoning was positively related to HDL. We also found that in the APOE $\varepsilon 3$ group, elevated $B G$ was associated with cognitive decline in terms of immediate memory, executive function, and perceptual reasoning. HDL was positively associated with executive function. In the APOE $\varepsilon 4$ group, higher BG was also correlated with a decline in abstract reasoning, represented by WMVis-I performance. In the APOE $\varepsilon 2$ group, BG was not related to cognitive function.

Some researchers have proposed that AD is a third type of diabetes that involves the central nervous system [25], and it is therefore interesting to determine the association between AD and glucose levels. Previous studies have indicated that diabetes or elevated BG exerts a specific influence on cognitive decline in dementia-free older adults $[6,26]$. In this study, we found that elderly adults with higher BG had poorer cognitive performance in terms of immediate memory and executive function, consistent with reports from the Shanghai sampling study [5] and a multiethnic elderly cohort report [27]. Although many studies have reported that higher BG or diabetes was a risk factor of dementia, the results vary among different groups of people. Several reports from community-based studies have shown that individuals with high BG had lower scores in processing speed, executive function [8], and delayed memory [28]. Many cross-sectional studies have found no relationship between fasting blood glucose levels and cognitive function in people with type 2 diabetes without dementia [29]. The inconsistent results in terms of the different cognitive domains might be attributable to the age and sample of participants, as well as the cognitive assessment method. Our finding showed that high blood glucose was related to cognitive decline based on the RAVL-I and COWAT scores. RAVL-I not only assessed immediate memory but also attention and verbal reasoning. In addition to the executive function domain, COWAT also evaluated verbal ability. Since high BG is related to atrophy in the hippocampus [30], it is not surprising that high BG was related to working memory and verbal ability. In rat research, high BG has been shown to impair hippocampal synaptic plasticity [31]. BG was one component of the 7-point scale proposed by the American Heart Association. The Framingham cohort has shown that ideal cardiovascular health was negatively associated with vascular dementia, cognitive decline on visual memory and reasoning, and frontal brain atrophy [32]. It suggested that cardiovascular and metabolic health are closely linked to brain health.

Reitz et al. showed that dysglycemia was associated with lower performance in language, speed, and visuospatial function [27]; however, these associations were attenuated when adjustment was made for APOE $\varepsilon 4$ and other vascular risk factors. This may indicate that the effects of elevated BG on cognitive impairment vary in the different APOE genotype groups. APOE $\varepsilon 3$ was previously considered to be a neutral gene, but our previous study showed that APOE $\varepsilon 3 / \varepsilon 3$ exhibited high blood glucose levels in Chinese non-demented older adults [16]. It is known that $\mathrm{APOE} \varepsilon 3$ is the most common genotype. In the current study, worse cognitive performance on immediate memory and executive function tests were significantly associated with higher BG levels, and these correlations were mainly manifested in the APOE $\varepsilon 3$ group. This might suggest that $\mathrm{APOE} \varepsilon 3$ is a functional gene with different effects on cognition. In our study, an association was found between BG and COWAT in the APOE $\varepsilon 3$ group, but not in the APOE $\varepsilon 4$ group, which is inconsistent with earlier findings of an association of BG with verbal memory [33, 34]. We also found that $\mathrm{APOE} \varepsilon 4$ genotype carriers' cognitive function in terms of abstract reasoning, but no other cognitive domains, was related to BG levels, which is partly consistent with the findings of a previous study [35, 36], but inconsistent with other research results [37]. This finding might be due to the small sample of APOE $\varepsilon 4$ carriers in this study, and the age of the APOE $\varepsilon 4$ group was younger than the other two groups. APOE $\varepsilon 4$ is considered an established risk gene of late-onset $\mathrm{AD}$. The main factors that cause cognitive decline in APOE $\varepsilon 4$ carriers may not relate to BG. In the APOE $\varepsilon 2$ group, BG was not associated with cognition and it may be that the protection offered by the $\varepsilon 2$ allele offset the negative influence of BG on cognition.

Cholesterol plays an important role in cognitive function [38]. APOE genotypes are associated with cholesterol 
levels [39]. In our study, HDL was associated with the block design test scores in the APOE $\varepsilon 3$ group and TG was associated with WMVis-I scores in the APOE $\varepsilon 4$ group. This indicates that the APOE genotype might affect cognitive function via cholesterol or triglycerides.

Although some studies have demonstrated a significant association between diabetes and the severity of deep WMHs [40], others have shown no such association [41] or non-significant trends [5]. We showed that higher BG was associated with more severe deep WMH, but there were no statistically significant differences between the high (abnormal) fasting BG and normal fasting BG groups. A previous review had reported that diabetes was not associated with WMH progression [42]. However, the APOE $\varepsilon 4$ risk allele was associated with higher WMH [43]. In our study, only a small sample had MRI data, and thus, the effect of higher BG on WMH in individuals with the APOE $\varepsilon 4$ genotype carriers could not be addressed.

In addition to the small sample size, there are some other limitations in our study. This was a cross-sectional study, and the long-term effects of BG on cognition cannot be inferred. Furthermore, we only had data on fasting BG, which could not provide information on insulin sensitivity, and we did not assess hemoglobin A1c levels. In addition, in the statistical analyses, we did not exclude people with diabetes because individual history of this disease was self-reported by participants; however, this may have biased the results. Lastly, the ratings of white matter lesions were qualitatively assessed by a neurologist and not by quantifiable methods. Further research should consider expanding sample testing using MRI data so as to quantify any differences.

\section{Conclusion}

Our results showed that older adults in a Chinese community who had high BG levels performed worse in various cognitive domains when they were APOE $\varepsilon 3 / \varepsilon 3$ carriers, compared with the performance of those who carried the APOE $\varepsilon 4$ or $\varepsilon 2$ allele. High BG might also be related to WMH. The main clinical implication of our findings is that high BG also increases the risk of cognitive impairment, even among the "neutral" APOE $\varepsilon 3 / \varepsilon 3$ genotype carriers. High BG may impact brain microvasculopathy in older adults. Thus, controlling BG in older individuals may reduce the risk of cognitive impairment. The need for interventions may be assessed according to the different APOE genotypes.

\section{Abbreviations}

AD: Alzheimer's disease; APOE: Apolipoprotein E; BG: Blood glucose; BMI: Body Mass Index; CLAS: China Longitudinal Ageing Study; COWAT: Controlled Word Association Test; DWMH: Deep white matter lesion hyperintensity; HDL-C: High destiny lipoprotein cholesterol; LDL-C: Low destiny lipoprotein cholesterol; MoCA: Montreal Cognitive Assessment; MRI: Magnetic resonance imaging; NTB: Neuropsychological Test Battery;
PCR: Polymerase chain reaction; PWMH: Periventricular lesion hyperintensity; RAVL-D: Rey Auditory Verbal Learning Delayed; RAVL-I: Rey Auditory Verbal Learning-Immediate; SCID: Structured Clinical Interview for DSM-IV; TC: Total cholesterol; TG: Triglycerides; WAIS-BD: Wechsler Adults Intelligence Scale Block Design; WAIS-PC: Wechsler Adults Intelligence Scale - Picture Completion; WMDS: Wechsler Memory-Digit Span; WMH: White matter hyperintensity; WMVis-I: Wechsler Memory Visual-Immediate

\section{Acknowledgments}

We wish to thank Li Zhong, Zhenlian Zhang, and Zhe Wang from Xiamen University for APOE genotyping, and all the participants in the study. We would like to thank Editage [https://www.editage.cn/] for English language editing.

\section{Funding}

This work was supported by grants from the National Key R\&D Program of China (No.2017YFC1310500), National Natural Science Foundation of China (No.81671402), and the Shanghai Science\& Technology Committee (No.15411961400)

\section{Availability of data and materials}

The datasets used and/or analysed during the current study are available from the corresponding author on reasonable request.

\section{Authors' contributions}

$X \mathrm{~L}$ and S-FX contributed to the study concept and design. LS, M-JZ, TW, J$\mathrm{HW}$, and G-JL acquired the data. QQ and XL analyzed the data and drafted the manuscript. All authors read and approved the final manuscript.

Ethics approval and consent to participate

All the subjects signed an informed consent form at the start of the study, and ethical approval was obtained from Shanghai Mental Health Center.

Consent for publication

Not applicable.

\section{Competing interests}

The authors declare that they have no competing interests.

Received: 15 June 2018 Accepted: 18 March 2019

Published online: 03 April 2019

\section{References}

1. Patterson C. World Alzheimer Report 2018 The state of the art of dementia research: New frontiers. Alzheimer's Disease International. 2018. [Available from: https://www.alz.co.uk/research/WorldAlzheimerReport2018.pdf].

2. Crichton GE, Elias MF, Davey A, Sullivan KJ, Robbins MA. Higher HDL cholesterol is associated with better cognitive function: the Maine-Syracuse study. J Int Neuropsychol Soc. 2014;20(10):961-70.

3. Corley J, Starr JM, Deary IJ. Serum cholesterol and cognitive functions: the Lothian birth cohort 1936. Int Psychogeriatr. 2015;27(3):439-53.

4. Livingston G, Sommerlad A, Orgeta V, Costafreda SG, Huntley J, Ames D, et al. Dementia prevention, intervention, and care. Lancet. 2017;390(10113): 2673-734.

5. Mortimer JA, Borenstein AR, Ding D, DeCarli C, Zhao Q, Copenhaver C, et al. High normal fasting blood glucose is associated with dementia in Chinese elderly. Alzheimers Dement. 2010;6(6):440-7.

6. Seetharaman S, Andel R, McEvoy C, Dahl Aslan AK, Finkel D, Pedersen NL. Blood glucose, diet-based glycemic load and cognitive aging among dementia-free older adults. J Gerontol A. 2015;70(4):471-9.

7. Vernooij MW, Ikram M, Vrooman HA, et al. White matter microstructural integrity and cognitive function in a general elderly population. Arch Gen Psychiatry. 2009;66(5):545-53

8. Qiu C, Sigurdsson S, Zhang Q, Jonsdottir MK, Kjartansson O, Eiriksdottir G, et al. Diabetes, markers of brain pathology and cognitive function. Ann Neurol. 2014;75(1):138-46

9. Yaffe K. Metabolic syndrome and cognitive disorders: is the sum greater than its parts? Alzheimer Dis Assoc Disord. 2007;21(2):167-71.

10. Mahley RW, Stanley C, Rall J, Apolipoprotein E. Far more than a lipid transport protein. Annu Rev Genomics Hum Genet. 2000;1(1):507-37. 
11. Davignon J, Gregg RE, Sing CF. Apolipoprotein E polymorphism and atherosclerosis. Arteriosclerosis. 1988;8(1):1-21.

12. Chuang YF, Hayden KM, Norton MC, Tschanz J, Breitner JCS, Welsh-Bohmer KA, et al. Association between APOE $\varepsilon 4$ allele and vascular dementia: the Cache County study. Dement Geriatr Cogn Disord. 2010;29(3):248-53.

13. Huang Y-WA, Zhou B, Wernig M, Südhof TC. ApoE2, ApoE3, and ApoE4 differentially stimulate APP transcription and AB secretion. Cell. 2017; 168(3):427-41.e21.

14. Leduc V, Jasmin-Bélanger S, Poirier J. APOE and cholesterol homeostasis in Alzheimer's disease. Trends Mol Med. 2010;16(10):469-77.

15. Martins IJ, Hone E, Foster JK, Sünram-Lea SI, Gnjec A, Fuller SJ, et al. Apolipoprotein $\mathrm{E}$, cholesterol metabolism, diabetes, and the convergence of risk factors for Alzheimer's disease and cardiovascular disease. Mol Psychiatry. 2006;11:721.

16. Ban C-X, Zhong L, Wang T, Zhu M-J, Wang J-H, Zhang Z-L, et al. Enhanced diabetes susceptibility in community dwelling Han elders carrying the Apolipoprotein E 3/3 genotype. PLoS One. 2016;11(3):e0151336.

17. Dore GA, Elias MF, Robbins MA, Elias PK, Nagy Z. Presence of the APOE $\varepsilon 4$ allele modifies the relationship between type 2 diabetes and cognitive performance: the Maine-Syracuse study. Diabetologia. 2009;52(12):2551-60.

18. Bangen KJ, Beiser A, Delano-Wood L, Nation DA, Lamar M, Libon DJ, et al. APOE genotype modifies the relationship between midlife vascular risk factors and later cognitive decline. J Stroke Cerebrovasc Dis. 2013;22(8):1361-9.

19. Irie F, Fitzpatrick AL, Lopez OL, et al. Enhanced risk for alzheimer disease in persons with type 2 diabetes and apoe $\varepsilon 4$ : the cardiovascular health study cognition study. Arch Neurol. 2008;65(1):89-93.

20. Xiao S, Li J, Tang M, Chen W, Bao F, Wang H, et al. Methodology of China's national study on the evaluation, early recognition, and treatment of psychological problems in the elderly: the China longitudinal aging study (CLAS). Shanghai Arch Psychiatry. 2013;25(2):91-8.

21. Zhong L, Xie Y-Z, Cao T-T, Wang Z, Wang T, Li X, et al. A rapid and costeffective method for genotyping apolipoprotein E gene polymorphism. Mol Neurodegener. 2016;11(1):2.

22. Nasreddine ZS, Phillips NA, Bedirian V, Charbonneau S, Whitehead V, Collin et al. The Montreal cognitive assessment, MoCA: a brief screening tool for mild cognitive impairment. J Am Geriatr Soc. 2005;53(4):695-9.

23. Gilman S, Koller M, Black R, Jenkins L, Griffith S, Fox N, et al. Clinical effects of $A \beta$ immunization (AN1792) in patients with $A D$ in an interrupted trial. Neurology. 2005;64(9):1553-62.

24. Fazekas F, Chawluk JB, Alavi A, Hurtig HI, Zimmerman RA. MR signal abnormalities at 1.5 T in Alzheimer's dementia and normal aging. AJR Am J Roentgenol. 1987;149(2):351-6.

25. de la Monte SM, Wands JR. Alzheimer's disease is type 3 diabetes_evidence reviewed. J Diabetes Sci Technol. 2008;2(6):1101-13.

26. Rajan KB, Arvanitakis Z, Lynch EB, McAninch EA, Wilson RS, Weuve J, et al. Cognitive decline following incident and preexisting diabetes mellitus in a population sample. Neurology. 2016;87(16):1681-7.

27. Reitz C, Guzman VA, Narkhede A, DeCarli C, Brickman AM, Luchsinger JA. Relation of Dysglycemia to structural brain changes in a multiethnic elderly cohort. J Am Geriatr Soc. 2017;65(2):277-85.

28. Kerti L, Witte AV, Winkler A, Grittner U, Rujescu D, Flöel A. Higher glucose levels associated with lower memory and reduced hippocampal microstructure. Neurology. 2013;81(20):1746-52.

29. Geijselaers SLC, Sep SJS, Stehouwer CDA, Biessels GJ. Glucose regulation, cognition, and brain MRI in type 2 diabetes: a systematic review. Lancet Diabetes Endocrinol. 2015;3(1):75-89.

30. Cherbuin N, Sachdev P, Anstey KJ. Higher normal fasting plasma glucose is associated with hippocampal atrophy: the PATH study. Neurology. 2012; 79(10):1019-26.

31. Stranahan AM, Norman ED, Lee K, Cutler RG, Telljohann RS, Egan JM, et al. Diet-induced insulin resistance impairs hippocampal synaptic plasticity and cognition in middle-aged rats. Hippocampus. 2008;18(11):1085-8.

32. Pase MP, Beiser A, Enserro D, Xanthakis V, Aparicio H, Satizabal CL, et al. Association of Ideal Cardiovascular Health with Vascular Brain Injury and Incident Dementia. Stroke. 2016;47(5):1201-6.

33. Ekblad LL, Rinne JO, Puukka PJ, Laine HK, Ahtiluoto SE, Sulkava RO, et al. Insulin resistance is associated with poorer verbal fluency performance in women. Diabetologia. 2015;58(11):2545-53.

34. Reger MA, Watson GS, Frey WH, Baker LD, Cholerton B, Keeling ML, et al. Effects of intranasal insulin on cognition in memory-impaired older adults: modulation by APOE genotype. Neurobiol Aging. 2006;27(3):451-8.
35. Claxton A, Baker LD, Hanson A, Trittschuh EH, Cholerton B, Morgan A, et al. Long-acting intranasal insulin detemir improves cognition for adults with mild cognitive impairment or early-stage Alzheimer's disease dementia. J Alzheimers Dis. 2015:44(3):897-906.

36. Ravona-Springer R, Heymann A, Schmeidler J, Sano M, Preiss R, Koifman K, et al. The ApoE4 genotype modifies the relationship of long-term glycemic control with cognitive functioning in elderly with type 2 diabetes. Eur Neuropsychopharmacol. 2014;24(8):1303-8.

37. Zhen J, Lin T, Huang $X$, Zhang H, Dong S, Wu Y, et al. Association of ApoE genetic polymorphism and type 2 diabetes with cognition in nondemented aging Chinese adults: a community based cross-sectional study. Aging Dis. 2018;9(3):346-57.

38. Frisardi V, Solfrizzi V, Seripa D, Capurso C, Santamato A, Sancarlo D, et al. Metabolic-cognitive syndrome: a cross-talk between metabolic syndrome and Alzheimer's disease. Ageing Res Rev. 2010;9(4):399-417.

39. Tao QQ, Chen Y, Liu ZJ, Sun YM, Yang P, Lu SJ, et al. Associations between apolipoprotein $\mathrm{E}$ genotypes and serum levels of glucose, cholesterol, and triglycerides in a cognitively normal aging Han Chinese population. Clin Interv Aging. 2014;9:1063-7.

40. Tamura Y, Kimbara Y, Yamaoka T, Sato K, Tsuboi Y, Kodera R, et al. White matter Hyperintensity in elderly patients with diabetes mellitus is associated with cognitive impairment, functional disability, and a high Glycoalbumin/ Glycohemoglobin ratio. Front Aging Neurosci. 2017;9:220.

41. Verdelho A, Madureira S, Ferro JM, Basile A-M, Chabriat H, Erkinjuntti T, et al. Differential impact of cerebral white matter changes, diabetes, hypertension and stroke on cognitive performance among non-disabled elderly. The LADIS study. J Neurol Neurosurg Psychiatry. 2007;78(12):1325-30.

42. Prins ND, Scheltens P. White matter hyperintensities, cognitive impairment and dementia: an update. Nat Rev Neurol. 2015;11:157.

43. Brickman AM, Schupf N, Manly JJ, Stern Y, Luchsinger JA, Provenzano $\mathrm{FA}$, et al. APOE $\varepsilon 4$ and risk for Alzheimer's disease: do regionally distributed white matter hyperintensities play a role? Alzheimers Dement. 2014;10(6):619-29.

\section{Ready to submit your research? Choose BMC and benefit from:}

- fast, convenient online submission

- thorough peer review by experienced researchers in your field

- rapid publication on acceptance

- support for research data, including large and complex data types

- gold Open Access which fosters wider collaboration and increased citations

- maximum visibility for your research: over $100 \mathrm{M}$ website views per year

At BMC, research is always in progress.

Learn more biomedcentral.com/submissions 